\title{
From Local Volatility to Local Lévy Models
}

\author{
Peter Carr \\ Courant Institute, New York University \\ Hélyette Geman \\ Université Paris-Dauphine and ESSEC \\ Dilip B. Madan \\ Robert H Smith School of Business \\ University of Maryland \\ Marc Yor \\ Laboratoire de Probabilités et Modèles aléatoires \\ Université Paris VI \& Université Paris VII.
}

February 292004

\begin{abstract}
We define the class of local Lévy processes. These are Lévy processes time changed by an inhomogeneous local speed function. The local speed function is a deterministic function of time and the level of the process itself. We show how to reverse engineer the local speed function from traded option prices of all strikes and maturities. The local Lévy processes generalize the class of local volatility models. Closed forms for local speed functions for a variety of cases are also presented. Numerical methods for recovery are also described.
\end{abstract}

\section{Introduction}

Local volatility models (Dupire (1994), Derman and Kani (1994)), were developed as a class of one dimensional Markov models with continuous sample paths that reprice all the traded European options. These models generalize the Black and Scholes (1973) and Merton (1973) models by making the instantaneous volatility of the stock returns a deterministic function of time and the stock price. Such a function is called the local volatility function. The risk neutral dynamics is fully specified on setting the growth rate of the stock at the instantaneous interest rate less the dividend yield. The resulting model is widely used for pricing contingent claims written on the stock price, including a variety of path dependent options. 
The local uncertainty of a local volatility model is Gaussian with zero skewness and kurtosis equal to 3 . It seems desirable in this context to accomodate a local uncertainty that allows for both skewness and excess levels of kurtosis. Many researchers have already noted for a variety of purposes, that one should introduce the possibility of jumps (Bakshi, Cao and Chen (1999), Bates (1996)). We have argued in prior research that the use of a jump process with infinite activity, i.e. one allowing infinitely many jumps in any time interval, effectively subsumes the need for an additional diffusion component (Carr, Geman, Madan and Yor (2002)). We therefore replace the local diffusive risk neutral dynamics by a local exposure to a Lévy process. This class of processes is increasingly being used in the study of financial market prices (Eberlein, Keller and Prause (1998), Barndorff-Nielsen and Shephard (2001), Geman, Madan and Yor (2001), Eberlein, Kallsen, Kristen (2003)).

Lévy processes offer a wide class of candidates for an alternative representation. We wish to formulate in this paper a class of local Lévy models that also reprice all the traded European options and provide a richer risk neutral dynamics.

We view the local volatility model in its equivalent formulation modeling $\log$ prices as Brownian motion running at the speed of the square of the local volatility function. Our essential idea is to replace Brownian motion with a Lévy process running at what we call the local speed function. Our local speed function is still a deterministic function of the level of the stock price and time. The Lévy process involved in this procedure is fixed through time and it only its speed that is space time dependent. This generalizes the role of Brownian motion, a particular Lévy process, in the local volatility model.

In a direct analogy with the contribution of the local volatility model, we show how to recover the local speed function from quoted option prices. Our final results are comparable to local volatility models, except that we employ a transform of the calendar spread in place of the calendar spread to infer the speed function.

We provide some explicit examples associated with particular local Lévy models permitting closed form recovery of local speed functions from option prices. The recovery function can in these cases be seen as a direct generalization of the comparable result for local volatility models. For other Lévy processes we describe procedures for numerical solutions, that still permit an efficient recovery of the local speed function. We also consider the "arithmetic" (Bachelier) case where options are written directly on a martingale, as opposed to a positive, exponential martingale. These results could be of financial interest in markets for options written on the profit and loss distribution of a portfolio of hedge funds directly.

The outline of the paper is as follows. Section 2 presents the details of the one dimensional Markov model describing the risk neutral dynamics for the discounted asset price and presents the general integral equation to be solved for recovery of the local speed function. The derivation for the local speed recovery procedure is given in section 3. In section 4 we consider a specific local Lévy process permitting closed form recovery. The arithmetic case is developed in 
section 5 . Numerical procedures are presented in section 6 . Section 7 concludes.

\section{Local Lévy Models}

We begin by recalling briefly the local volatility model and the associated procedure for recovering the local volatility function from traded option prices. Let $S(t)$ denote the price of the stock at time $t, 0 \leq t \leq H$. Suppose the continuously compounded interest rate is $r$ and the dividend yield is $\eta$, also continuously compounded. The risk neutral dynamics for the stock price in the local volatility model is given by the following stochastic differential equation

$$
d S=(r-\eta) S d t+\sigma(S, t) S d W(t)
$$

where $W=(W(t), 0 \leq t \leq H)$ is a standard Brownian motion and $\sigma(S, t)$ is the local volatility function.

The relevance of the formulation (1) is quite extensive from the perspective of constructing Markov processes that match the marginals of general stochastic processes. Gyongy (1986) showed that one could associate with a general Ito process a one dimensional Markov process of the type (1) with a view to matching marginals. This question has also been studied from other perspectives in Madan and Yor (2002).

Let $C(K, T)$ denote the price at time zero, of a European call option of maturity $T$ and strike $K$. Dupire (1994), Derman and Kani (1994) showed that one may recover the local speed function from the prices of traded options using the formula

$$
\sigma(K, T)=2 \frac{C_{T}+\eta C+(r-\eta) K C_{K}}{K^{2} C_{K K}} .
$$

We generalize equation (1) by allowing for jumps in the stock price. We denote the size of the jump in the log price at any time by $x$. The Lévy measure $k(x) d x$ specifies the arrival rate of jumps of size per unit time. In analogy with the local volatility function, we introduce a local speed function $a(S, t)$ that measures the speed at which the Lévy process is running at time $t$ when the stock price is at the level $S$.

In addition to the exposure to the Brownian motion, our stock price process is also exposed to the compensated jump martingale with compensator

$$
\nu(d x, d u)=a(S(u), u) k(x) d x d u .
$$

The risk neutral dynamics for the stock price are now given by

$$
\begin{aligned}
d S= & (r-\eta) S\left(t_{-}\right) d t+\sigma\left(S\left(t_{-}\right), t\right) d W(t) \\
& +\int_{-\infty}^{\infty} S\left(t_{-}\right)\left(e^{x}-1\right)(m(d x, d u)-\nu(d x, d u))
\end{aligned}
$$

where $m(d x, d u)$ is the counting measure associated with the jumps in the logarithm of the stock price. 
The formulation of the compensator in (3) alters local volatility by running the Lévy process at a speed that is a deterministic function of the stock price and time. Alternatively, one could scale the jump sizes instead. In the case of Brownian motion, scaling and time changing are equivalent operations by the scaling property of Brownian motion, but for general Lévy processes these are different operations. Time changing leads to tractable results while scaling is much more complicated.

The objective of this paper is to show how one may recover the local speed function $a(S, t)$ from traded option prices in the context of a known local volatility function $\sigma(S, t)$. Of particular interest is the case of a pure jump process where $\sigma \equiv 0$ and the stock has no diffusion exposure. The solution for the local speed function employs in a critical way a convolution transform with the "double exponential tail" of the Lévy measure. We now define the double exponential tail of a Lévy measure.

We start by defining the double tail of a Lévy measure $k(x)$ as

$$
\psi(z)=\left\{\begin{array}{ll}
\int_{-\infty}^{z} \int_{-\infty}^{x} k(u) d u d x & z<0 \\
\int_{z}^{\infty} \int_{x}^{\infty} k(u) d u d x & z>0
\end{array} .\right.
$$

The double tail integrates the tail of the Lévy measure in both directions twice and hence we refer it to it as the double tail. It is important as it measures quadratic variation, which may be observed on applying integration by parts two times, and we have

$$
\int_{-\infty}^{\infty} \psi(z) d z=\frac{1}{2} \int_{-\infty}^{\infty} x^{2} k(x) d x .
$$

The double exponential tail $\psi_{e}(z)$ employs an exponential weighting and we have

$$
\psi_{e}(z)=\left\{\begin{array}{cc}
\int_{-\infty}^{z} e^{x} \int_{-\infty}^{x} k(u) d u & z<0 \\
\int_{z}^{\infty} e^{x} \int_{x}^{\infty} k(u) d u & z>0
\end{array}\right.
$$

Equivalently one may write

$$
\psi_{e}(z)=\left\{\begin{array}{cc}
\int_{-\infty}^{z}\left(e^{z}-e^{x}\right) k(x) d x & z<0 \\
\int_{z}^{\infty}\left(e^{x}-e^{z}\right) k(x) d x & z>0
\end{array} .\right.
$$

The exponential double tail may be viewed as the price of instantaneous outof-the-money call and put options struck at $e^{z}$.

The solution for the local speed function is

$$
a(K, T)=\frac{b(\ln (K), T)}{K^{2} C_{K K}}
$$


where the convolution transform of $b$ with the exponential double tail is

$$
\begin{aligned}
\int_{-\infty}^{\infty} b(y, T) \psi_{e}(k-y) d y= & c_{T}+\eta c+\left(r-\eta+\frac{\sigma^{2}\left(e^{k}, T\right)}{2}\right) c_{k}-\frac{\sigma^{2}\left(e^{k}, T\right)}{2} c_{k k} \\
& k \underset{D e f}{=} \ln (K) \\
& c(k, T) \underset{D e f}{=} C\left(e^{k}, T\right) .
\end{aligned}
$$

We see that in equation (7) the local speed function is related to calendar spreads and butterfly spread prices, much as it is in the local volatility case, except that we have a convolution integral of the effective function with the double exponential tail replacing the direct use of the calendar spread. This spreading occurs to account for the distribution of the jump sizes across the real line.

The solution of equation (7) for the local speed function $a(S, t)$ requires a prior specification of the local volatility component $\sigma(S, t)$. In the special case when this is zero and we have a pure jump process the convolution of $b$ and $\psi$ is comparable to the use of the calendar spread in the recovery of local volatility. In both cases one is essentially retrieving the local quadratic variation as a measure of the speed.

\section{Recovering Local Speed Functions from Op- tion Prices}

The integrated form of the risk neutral stock price dynamics of equation (4) may be written in the form

$$
\begin{aligned}
S(t)= & S(0)+\int_{0}^{t} S\left(u_{-}\right)(r-\eta) d u \\
& +\int_{0}^{t} S\left(u_{-}\right) \sigma(S(u), u) d W(u) \\
& +\int_{0}^{t} \int_{-\infty}^{\infty} S\left(u_{-}\right)\left(e^{x}-1\right)\left(m(d x, d u)-\nu_{X}(d x, d u)\right)
\end{aligned}
$$

Note that the drift for the stock return is indeed $r-\eta$, and the martingale terms admit both continuous and jump components. This decomposition is useful in evaluating expectations of functions of the stock price, like a call option payoff. We shall in particular employ a generalization of Ito's lem ma to convex functions known as the Meyer-Tanaka formula (see for example Meyer (1976), DellacherieMeyer (1980), and Yor (1978) for the specific formulation below). In particular, 
for the call option payoff at maturity we have

$$
\begin{aligned}
(S(T)-K)^{+}= & (S(0)-K)^{+}+\int_{0}^{T} \mathbf{1}_{S(u-)>K} d S(u) \\
& \left.+\frac{1}{2} \int_{0}^{T} \mathbf{1}_{(S(u)=K)^{2}} \sigma^{2} S(u), u\right) S^{2}(u) d u \\
& +\sum_{u \leq T} \mathbf{1}_{S(u-)>K}(K-S(u))^{+}+\mathbf{1}_{S(u-)<K}(S(u)-K)^{+}
\end{aligned}
$$

We see, in the case of zero interest rates and dividend yields, that the payoff to the call option is made up of intrinsic value and a time value represented by the value of last two terms (the second term, in this case has zero value as the stock is a martingale). The second integral denotes the value at $K$ of the continuous local time $L_{T}^{a} ; a \in \mathbb{R}$, which is globally defined as $\int_{-\infty}^{\infty} f(a) L_{T}^{a} d a=$ $\int_{0}^{T} f\left(S\left(u_{-}\right) d\left\langle S^{c}\right\rangle_{u}\right.$ where $d\left\langle S^{c}\right\rangle_{u}=\sigma^{2}(S(u), u) S^{2}(u) d u$, and is here applied formally to the Dirac measure $f(a)=\delta_{K}(a)$. The discontinuous component of local time is made up of just the crossovers whereby one receives $S(u)-K$ on crossing the strike into the money while one receives $(K-S(u))$ on crossing the strike out of the money.

The next step is to compute expectations on both sides of (9). For this we introduce $q(\Sigma, u)$ the transition density that the stock price is $\Sigma$ at time $u$ given that at time 0 it is at $S(0)$. We may write the expectation of the equation (9) in terms of the call price function and the function $q(Y, u)$ as

$$
\begin{aligned}
e^{r T} C(K, T)= & (S(0)-K)^{+}+\int_{0}^{T} \int_{K}^{\infty} d Y q(Y, u) Y(r-\eta) d u \\
& +\frac{1}{2} \int_{0}^{T} q(K, u) \sigma^{2}(K, u) K^{2} d u \\
& +\int_{0}^{T} \int_{K}^{\infty} d Y q(Y, u) \int_{-\infty}^{\ln \left(\frac{K}{Y}\right)}\left(K-Y e^{x}\right) \nu_{X}(d x, d u) \\
& +\int_{0}^{T} \int_{0}^{K} d Y q(Y, u) \int_{\ln \left(\frac{K}{Y}\right)}^{\infty}\left(Y e^{x}-K\right) \nu_{X}(d x, d u)
\end{aligned}
$$

We now specialize our Lévy system to that of a time changed Lévy process as described in equation (3). In this case we obtain

$$
\begin{aligned}
e^{r T} C(K, T)= & (S(0)-K)^{+}+\int_{0}^{T} \int_{K}^{\infty} d Y Y q(Y, u)(r-\eta) d u \\
& +\frac{1}{2} \int_{0}^{T} q(K, u) \sigma^{2}(K, u) K^{2} d u \\
& +\int_{0}^{T} \int_{K}^{\infty} d Y q(Y, u) a(Y, u) \int_{-\infty}^{\ln \left(\frac{K}{Y}\right)}\left(K-Y e^{x}\right) k(x) d x d u \\
& +\int_{0}^{T} \int_{0}^{K} d Y q(Y, u) a(Y, u) \int_{\ln \left(\frac{K}{Y}\right)}^{\infty}\left(Y e^{x}-K\right) k(x) d x d u
\end{aligned}
$$


Finally differentiating the equation (10) with respect to $T$ we get

$$
\begin{aligned}
r e^{r T} C+e^{r T} C_{T}= & (r-\eta) \int_{K}^{\infty} q(Y, T) Y d Y \\
& +\frac{\sigma^{2}(K, T) K^{2}}{2} q(K, T) \\
& +\int_{K}^{\infty} d Y Y q(Y, T) \int_{-\infty}^{\ln \left(\frac{K}{Y}\right)}\left(e^{\ln \left(\frac{K}{Y}\right)}-e^{x}\right) \nu_{X}(d x, d u) \\
& +\int_{0}^{K} d Y Y q(Y, T) \int_{\ln \left(\frac{K}{Y}\right)}^{\infty}\left(e^{x}-e^{\ln \left(\frac{K}{Y}\right)}\right) \nu_{X}(d x, d u)
\end{aligned}
$$

We now solve for $C_{T}$, noting some elementary properties of the relationship between call prices and the risk neutral density. In particular we note

$$
\begin{aligned}
e^{-r T} \int_{0}^{\infty} Y q(Y, T) d Y & =C-K C_{K} \\
e^{-r T} q(K, T) & =C_{K K}
\end{aligned}
$$

Solving for $C_{T}$ we get that

$$
\begin{aligned}
C_{T}= & -\eta C-(r-\eta) K C_{K}+\frac{\sigma^{2}(K, T) K^{2}}{2} C_{K K} \\
& +\int_{K}^{\infty} d Y Y C_{Y Y} a(Y, T) \int_{-\infty}^{\ln \left(\frac{K}{Y}\right)}\left(e^{\ln \left(\frac{K}{Y}\right)}-e^{x}\right) k(x) d x \\
& +\int_{0}^{K} d Y Y C_{Y Y} a(Y, T) \int_{\ln \left(\frac{K}{Y}\right)}^{\infty}\left(e^{x}-e^{\ln \left(\frac{K}{Y}\right)}\right) k(x) d x .
\end{aligned}
$$

We now recognize the double exponential tail in the integral terms. In terms of this exponential double tail we may write the calendar spread value, $C_{T}$, as

$$
\begin{aligned}
C_{T}= & -\eta C-(r-\eta) K C_{K}+\frac{\sigma^{2}(K, T) K^{2}}{2} C_{K K} \\
& +\int_{0}^{\infty} C_{Y Y} Y a(Y, T) \psi\left(\ln \left(\frac{K}{Y}\right)\right) d Y
\end{aligned}
$$

When there are no jumps in the process for $X$ and $\psi \equiv 0$, equation (12) is identical to the equation employed in inferring local volatilities from market call prices. In the opposite case, when there is no continuous martingale component we have the result

$$
C_{T}+\eta C+(r-\eta) K C_{K}=\int_{0}^{\infty} C_{Y Y} Y a(Y, T) \psi\left(\ln \left(\frac{K}{Y}\right)\right) d Y
$$

It is now useful to rewrite equation (13) in terms of $k=\ln (K), y=\ln (Y)$ 
and $c(k, T)=C\left(e^{k}, T\right)$. With this substitution we may rewrite (12) as

$$
\begin{aligned}
& c_{T}+\eta c+\left(r-\eta+\frac{\sigma^{2}\left(e^{k}, T\right)}{2}\right) c_{k}-\frac{\sigma^{2}\left(e^{k}, T\right)}{2} c_{k k} \\
= & \int_{-\infty}^{\infty} b(y, T) \psi(k-y) d y \\
\text { where } b(y, T)= & e^{2 y} C_{Y Y}\left(e^{y}, T\right) a\left(e^{y}, T\right)
\end{aligned}
$$

The forward speed function, $a(Y, T)$, may be identified as

$$
a(Y, T)=\frac{b(\ln (Y), T)}{Y^{2} C_{Y Y}} .
$$

We may identify from the convolution transform (14) with the exponential double tail the function $b(y, T)$ at each maturity using data on option prices. Equation (15) then determines the forward speed function for the local Lévy model. For specific Lévy measures the convolution equation (14) may be solved in closed form to yield explicit solutions for the Markov process from data on option prices. The next section presents such examples.

\section{Closed form local Lévy models}

The section presents an example of an explicit expressions for the local speed function in terms of the derivatives of the call price function. The result is obtained for a specific class of driving Lévy processes and generalize similar expressions known for local volatility. The solution method relies on recognizing the inverse of the convolution operator in our convolution transform equation.

We associate to a Lévy density its exponential double tail as defined by equation (6). We also associate with the exponential double tail, the convolution operator

$$
\Lambda_{\psi}: f \rightarrow \Lambda_{\psi}(f)
$$

where

$$
\Lambda_{\psi}(f)(x)=\int_{-\infty}^{\infty} \psi(x-y) f(y) d y .
$$

Some discussion of such operators from an analytic point of view are found in Hirsch and Lacombe (1999). Our interest in identifying the forward speed function of the price process lies in inverting this operator.

It turns out that for certain Lévy measures $k, \Lambda_{\psi}$ is the resolvent operator $V_{\lambda}$ of a certain Lévy process $\left(Y_{t}, t \geq 0\right)$ with Lévy density $k$, for a given $\lambda$; more precisely

$$
\begin{aligned}
\Lambda_{\psi}(f)(x) & =c V_{\lambda}(f)(x) \\
& \equiv c E_{x}\left[\int_{0}^{\infty} e^{-\lambda t} f\left(Y_{t}\right) d t\right] .
\end{aligned}
$$


Denote by $p_{t}(x-y)$ the density of $Y_{t}$ under $\mathbb{P}_{x}$; then if equation (16) holds (See, Sato (1999), Bertoin (1998)) we have that

$$
\psi(x-y)=c \int_{0}^{\infty} d t e^{-\lambda t} p_{t}(x-y)
$$

i.e.

$$
\begin{aligned}
\psi(x) & =c \int_{0}^{\infty} d t e^{-\lambda t} p_{t}(x) \\
& \equiv c v_{\lambda}(x), x \in \mathbb{R} .
\end{aligned}
$$

Our interest in this situation comes from the fact that if equation (17) holds, then our convolution transform is related to the resolvent $V_{\lambda}$ by (16) and thanks to the relationship between the infinitesimal generator $A$ of $\left(Y_{t}\right)$ and its resolvent $\left(V_{\lambda}\right)$ we have:

$$
(\lambda I-A) V_{\lambda}=I .
$$

We recognize in equation (18) the inverse of our convolution transform operator as in general an integro-differential operator.

If we wish to solve

$$
\Lambda_{\psi}(f)=g
$$

then from equation (16)

$$
V_{\lambda}(f)=\frac{1}{c} g
$$

and from equation (18) we deduce that

$$
f=\frac{1}{c}(\lambda g-A g),
$$

that is we have inverted the convolution transform operator $\Lambda_{\psi}$. An example illustrates the details.

Consider the Lévy measure defined by the asymmetric negative exponential Lévy measure:

$$
k(x)=\left\{\begin{array}{cc}
\beta \exp (G x) & x<0 \\
\exp (-M x) & x>0
\end{array} .\right.
$$

where $G$ is positive and $M$ is greater than one. Such a jump component has been studied extensively in a financial context by Kou (2002) and Kou and Wang (2003). The double exponential tail of this Lévy measure is given by

$$
\psi(z)=\left\{\begin{array}{cc}
\frac{\beta \exp (-(G+1)|z|)}{G^{2}+G} & z<0 \\
\frac{\exp (-(M-1) z)}{M^{2}-M} & z>0
\end{array} .\right.
$$

On the other hand, let us consider $V_{\lambda}$ for $(B(u)+\mu u, u \geq 0)$, Brownian motion with drift $\mu$; we shall write $v_{\lambda}^{(\mu)}(x)$ for the resolvent density. We use the 
well known fact that, (see, e.g. Biane-Yor (1988) for discussions about the law of $\left.\left(B_{u}, u \leq T_{\theta^{2} / 2}\right)\right)$ if $T_{\theta^{2} / 2}$ denotes an exponential variable with parameter $\theta^{2} / 2$ independent from $(B(t), t \geq 0)$ then: $B\left(T_{\theta^{2} / 2}\right)$ has the Laplace distribution

$$
\frac{\theta}{2} \exp (-\theta|x|) d x \text {. }
$$

Hence it follows that

$$
\frac{\theta^{2}}{2} v_{\theta^{2} / 2}^{(0)}(x)=\frac{\theta}{2} \exp (-\theta|x|) .
$$

Equivalently we may write

$$
v_{\theta^{2} / 2}^{(0)}(x)=\frac{1}{\theta} \exp (-\theta|x|)
$$

We now compute $v_{\theta^{2} / 2}^{(\mu)}$, with the help of the Cameron-Martin relationship:

$$
\begin{aligned}
& \frac{\theta^{2}}{2} E\left[\int_{0}^{\infty} d t e^{-\frac{\theta^{2}}{2} t} f\left(B_{t}+\mu t\right)\right]=\frac{\theta^{2}}{2} E\left[\int_{0}^{\infty} d t e^{-\frac{\theta^{2}+\nu^{2}}{2} t} f\left(B_{t}\right) e^{\mu B_{t}}\right] \\
= & \left(\frac{\theta^{2}}{\nu^{2}}\right) \frac{\nu^{2}}{2} \int_{0}^{\infty} d t e^{-\frac{\nu^{2}}{2} t} E\left[f\left(B_{t}\right) e^{\mu B_{t}}\right], \nu^{2}=\theta^{2}+\mu^{2} \\
= & \left(\frac{\theta^{2}}{\nu^{2}}\right) \frac{\nu}{2} \int_{-\infty}^{\infty} f(x) e^{\mu x} e^{-\nu|x|} d x, \text { from }(21) \\
= & \left(\frac{\theta^{2}}{2 \nu}\right)\left\{\int_{-\infty}^{0} d x f(x) e^{-(\nu+\mu)|x|}+\int_{0}^{\infty} d x f(x) e^{-(\nu-\mu) x}\right\}
\end{aligned}
$$

It follows that

$$
v_{\theta^{2} / 2}^{(\mu)}(x)=\left\{\begin{array}{cc}
\frac{1}{\nu} \exp (-(\nu-\mu) x) & x>0 \\
\frac{1}{\nu} \exp (-(\nu+\mu) x) & x<0
\end{array}\right.
$$

which generalizes (21).

We now start with $\psi$ defined by equation (19) and determine $c,\left(\frac{\theta^{2}}{2}\right), \mu$ such that we have

$$
\psi(x)=c v_{\left(\theta^{2} / 2\right)}^{(\mu)}(x) .
$$

We must have

$$
\begin{aligned}
M-1 & =\nu-\mu \\
G+1 & =\nu+\mu
\end{aligned}
$$

It follows that

$$
\begin{aligned}
\nu & =\frac{G+M}{2} \\
\mu & =1+\frac{G-M}{2} \\
\theta^{2} & =\nu^{2}-\mu^{2}=M-1+(2 M-1) G
\end{aligned}
$$


We must also have

$$
\frac{1}{M^{2}-M}=\frac{\beta}{G^{2}+G}=\frac{c}{\nu}
$$

or

$$
c=\frac{\nu}{M^{2}-M}=\frac{G+M}{2\left(M^{2}-M\right)}
$$

We have to restrict the parameter $\beta$ in our Lévy measure by

$$
\beta=\frac{G^{2}+G}{M^{2}-M} .
$$

For this case we may write the solution for the forward speed function explicity as

$$
a(K, T)=\frac{\left(\frac{\theta^{2}}{2} I-\frac{1}{2} D^{2}-\mu D\right)\left(c_{T}+\eta c+(r-\eta) c_{k}\right)}{K^{2} C_{K K}}
$$

A particularly instructive case for comparison with the results for local volatility is when we take $r=\eta=0$. In this case we have

$$
\begin{aligned}
a(K, T) & =\frac{1}{K^{2} C_{K K}}\left(\frac{\theta^{2}}{2} C_{T}-\frac{1}{2} K^{2} C_{K K T}-(1+\mu) K C_{K T}\right) \\
& =\frac{\theta^{2} C_{T}}{2 K^{2} C_{K K}}-\frac{C_{K K T}}{2 C_{K K}}-\frac{(1+\mu) C_{K T}}{K C_{K K}}
\end{aligned}
$$

The formula (23) can be contrasted with results known for local volatility where we get

$$
\sigma^{2}(K, T)=\frac{2 C_{T}}{K^{2} C_{K K}}
$$

\section{The Arithmetic Case}

We develop in this section the results for recovery of the forward speed function from data on prices of options written on the level of a real valued martingale for various strikes and maturities. To distinguish the development from the previous section we use different notation and write the process for the underlying martingale $H$ as

$H(t)=H(0)+\int_{0}^{t} \zeta(H(u), u) d B(u)+\int_{0}^{t} \int_{-\infty}^{\infty} h(m(d h, d u)-a(H(u), u) w(h) d h d u)$

where $w(h)$ is a Lévy density for a base Lévy process that is time changed by the integral dependent on the past of the process $H$, in accordance with

$$
\int_{0}^{t} a(H(u), u) d u
$$


The volatility coefficient $\zeta$ is a deterministic function of the level of the martingale and calendar time and $B(t)$ is a standard Brownian motion.

We suppose that for real valued strikes, denoted by $L$, and for expiration dates, denoted by $T$, there are options trading at time 0 that payoff at time $T$ the value

$$
(H(T)-L)^{+}
$$

with current prices that we denote by $v(L, T)$.

Following the analysis of section 3 , and noting that here we work directly with expectations or forward prices, yields the result

$$
\begin{aligned}
(H(T)-K)^{+}= & (H(0)-L)^{+}+\int_{0}^{T} \mathbf{1}_{H(u-)>L} d H(u)+\frac{1}{2} \int_{0}^{T} \delta_{(H(u-)=L)} \frac{\zeta^{2}(u)}{2} d u \\
& +\int_{0}^{T} \mathbf{1}_{H\left(u_{-}\right)>L}(L-H(u))^{+} d u+\int_{0}^{T} \mathbf{1}_{H(u-)<L}(H(u)-L)^{+} d u .
\end{aligned}
$$

Taking expectations, and noting that since $H$ is a martingale, the second term will be zero, we get in terms of the transition densities $q(H, u)$ that

$$
\begin{aligned}
v(L, T)= & (H(0)-L)^{+}+\frac{1}{2} \int_{0}^{T} q(L, u) \zeta^{2}(L, u) d u \\
& +\int_{0}^{T} d u \int_{L}^{\infty} d H q(H, u) a(H, u) \int_{-\infty}^{L-H}(L-H-h) w(h) d h \\
& +\int_{0}^{T} d u \int_{-\infty}^{L} d H q(H, u) a(H, u) \int_{L-H}^{\infty}(H+h-L) w(h) d h .
\end{aligned}
$$

Differentiation with respect to maturity $T$ then yields

$$
\begin{aligned}
v_{T}= & q(L, T) \frac{\zeta^{2}(L, T)}{2} \\
& +\int_{L}^{\infty} d H q(H, T) a(H, T) \int_{-\infty}^{L-H}(L-H-h) w(h) d h \\
& +\int_{-\infty}^{L} d H q(H, T) a(H, T) \int_{L-H}^{\infty}(h-(L-H)) w(h) d h
\end{aligned}
$$

Introducing the function $\psi$ defined here by

$$
\psi(z)=\left\{\begin{array}{cc}
\int_{z}^{\infty}(h-z) w(h) d h & z>0 \\
\int_{-\infty}^{z}(z-h) w(h) d h & z<0
\end{array}\right.
$$

we may write that

$$
v_{T}=\frac{v_{L L}}{2} \zeta^{2}(L, T)+\int_{-\infty}^{\infty} d Z v_{Z Z} a(Z, T) \psi(L-Z) .
$$


The function $\psi$ is now seen to be just the double tail of the Lévy measure as defined in (5).

For the case where there are no jumps we get the well known result of local volatility models that

$$
\zeta^{2}(L, T)=\frac{2 v_{T}}{v_{L L}}
$$

Our interest is in the opposite case when $\zeta=0$ and $\psi$ is the double tail of a Lévy process. In this case we obtain the convolution transform equation

$$
v_{T}=\int_{-\infty}^{\infty} d Z v_{Z Z} a(Z, T) \psi(L-Z)
$$

that is to be solved for $a$.

We develop in particular the solution for the case of a symmetric, double exponential Lévy density

$$
w(h)=\theta \exp (-\theta|h|)
$$

for which the relevant $\psi$ function is

$$
\psi(z)=\frac{1}{\theta} \exp (-\theta|z|)
$$

that we recognize as the resolvent density of Brownian motion given by equation (21). It follows that the solution for $a$ is

$$
a(L, T)=\frac{1}{v_{L L}}\left(\lambda v_{T}-\frac{1}{2} v_{L L T}\right),
$$

where $\lambda=\theta^{2} / 2$.

\section{$6 \quad$ Numerical Procedures}

For more general Lévy processes in either the geometric or arithmetic case the basic convolution transform equations (14, and 24) may be solved numerically. Here we develop the procedures illustratively for the variance gamma Lévy process introduced in Madan, Carr and Chang (1998), and Carr, Geman, Madan and Yor (2002). The Lévy measure for this process is defined as $k_{V G}(x) d x$ where

$$
k_{V G}(x)=\left\{\begin{array}{cc}
\frac{\exp (-M x)}{x} & x>0 \\
\frac{\exp (-G|x|)}{|x|} & x<0
\end{array}\right.
$$

and the Lévy process is one of infinite activity that accomodates a negative skewness by taking $G>M$ and calibrates well option prices across all strikes, at any fixed maturity. 
In the geometric case, we denote the double exponential tail of the $V G$ Lévy measure by $\psi_{G V G}(z)$, and for the arithmetic case we denote the double tail by $\psi_{A V G}(z)$. On computation we obtain the following expressions for the requisite double tails.

$$
\psi_{G V G}(z)=\left\{\begin{array}{cc}
\int_{(M-1) z} \frac{e^{-u}}{u} d u-e^{z} \int_{M z} \frac{e^{-u}}{u} d u & z>0 \\
e^{-|z|} \int_{G|z|} \frac{e^{-u}}{u} d u-\int_{(G+1)|z|} \frac{e^{-u}}{u} d u & z<0
\end{array}\right.
$$

and

$$
\psi_{A V G}(z)=\left\{\begin{array}{cc}
\frac{e^{-M z}}{M}-z \int_{M z}^{\infty} \frac{e^{-u}}{u} d u & z>0 \\
\frac{e^{-G|z|}}{G}-|z| \int_{G|z|} \frac{e^{-u}}{u} d u & z<0
\end{array}\right.
$$

The basic equation to be solved is of the form

$$
\int_{-\infty}^{\infty} \psi(x-y) f(y)=g(x)
$$

where the function $g(x)$ is obtained from the data on option prices. We define the Fourier transforms of $f, g$ by

$$
\widehat{f}(\xi)=\int_{-\infty}^{\infty} e^{i \xi y} f(y) d y, \widehat{g}(\xi)=\int_{-\infty}^{\infty} e^{i \xi y} g(x) d x
$$

If the function $\psi(z)$ has a Fourier transform, $\widehat{\psi}$ then from the relationship of Fourier transforms to convolutions we have from equation (26a) that

$$
\widehat{f}(\xi)=\frac{\widehat{g}(\xi)}{\widehat{\psi}(\xi)}
$$

and we may obtain $f(y)$ using the inverse Fourier transform applied to $\widehat{f}(\xi)$.

The transform $\widehat{g}(\xi)$ is to numerically constructed from the calibration of market prices. However, in many cases of interest we may analytically obtain $\widehat{\psi}(\xi)$ the Fourier transform of the double exponential tail. We present here the result for the CGMY model studied in Carr, Geman, Madan and Yor (2002), that generalizes the variance gamma. In this case we obatin on integration that

$$
\begin{aligned}
\widehat{\psi}(\xi)= & \int_{-\infty}^{0} e^{i \xi x} \int_{-\infty}^{x}\left(e^{x}-e^{y}\right) C \frac{e^{-G|x|}}{|x|^{1+Y}} d x \\
& +\int_{0}^{\infty} e^{i \xi x} \int_{x}^{\infty}\left(e^{y}-e^{x}\right) C \frac{e^{-M x}}{x^{1+Y}} d x \\
= & \frac{\Gamma(-Y)}{i \xi(1+i \xi)}\left[\begin{array}{c}
(M-(1+i \xi))^{Y}-M^{Y} \\
(1+i \xi)\left((M-1)^{Y}-M^{Y}\right) \\
+(G+1+i \xi)^{Y}-G^{Y} \\
-(1+i \xi)\left((G+1)^{Y}-G^{Y}\right)
\end{array}\right]
\end{aligned}
$$

The final result follows by applying integration by parts twice to the integrals on the two sides. 


\section{Conclusion}

This paper defines the class of local Lévy processes as one dimensional Markov processes that are obtained by time changing a prespecified Lévy process. In practice one would choose the Lévy process with respect to its ability to explain short maturity call option prices across the strike domain. The specific time change is inhomogeneous and is given as the integral of a deterministic function of the price level and calendar time, called the local speed function. It is shown how this local speed function may be recovered from information on the prices of traded options of all strikes and maturities. In this regard, the paper generalizes the local volatility models to permit local dynamics that are capable of independently calibrating market skews. This reduces the burden on the volatility function in calibrating the model to data, and it is expected that such a move will produce more reasonable forward return distributions for the risk neutral asset returns.

For a variety of elementary cases, closed forms for the local speed function are presented in both the case of the exponential and arithmetic Lévy processes. For practical implementation, Fourier methods, already known to be highly successful in calibrating models to option data are extended here to the recovery of the local speed function from information on market implied volatilities across the maturity and strike dimensions. 


\section{References}

[1] Bakshi, G., C. Cao, and Z. Chen (1997), "Empirical Performance of Alternative Option Pricing Models," Journal of Finance, 52, 2003-2049.

[2] Barndorff-Nielsen, O. (1998), "Processes of Normal Inverse Gaussian type," Finance and Stochastics, 2, 41-68.

[3] Barndorff-Nielsen and N. Shephard (2001), "Non-Gaussian OrnsteinUhlenbeck-based models and some of their uses in financial economics (with discussion)," Journal of the Royal Statistical Society, Series B, 63, 167-241.

[4] Bates, D., 1996, "Jumps and Stochastic Volatility: Exchange Rate Processes Implicit in Deutschemark Options," Review of Financial Studies, 9, 69-108.

[5] Bertoin, J. (1998), Lévy Processes, Second Edition, Cambridge University Press, Cambridge.

[6] Biane-Yor (1988), "Sur la loi des temps locaux browniens pris en un temps exponentiel," Séminaire de Probabilités, XXII, Lecture Notes in Mathematics 1321, Springer-Verlag, Berlin, p. 454-466.

[7] Carr, P., H. Geman, D. Madan, and M. Yor (2002), "The Fine Structure of Asset Returns: An Empirical Investigation," Journal of Business, 75, 2, 305-332.

[8] Carr, P., H. Geman, D. Madan and M. Yor (2003), "Stochastic Volatility for Lévy Processes," Mathematical Finance, 13, 345-382.

[9] Dellacherie, C. and P. Meyer (1980), Probabilités et Potentiel, Theories des Martingales, Hermann, Paris.

[10] Derman, E. and I. Kani (1994), "Riding on a smile," Risk 7,32-39.

[11] Dupire, B. (1994), "Pricing with a smile," Risk 7, 18-20.

[12] Eberlein, E., U. Keller, and K. Prause (1998), "New Insights into Smile, Mispricing and Value at Risk," Journal of Business, 71, 371-406.

[13] Eberlein, E., J. Kallsen, J. Kristen (2003), "Risk Management Based On Stochastic Volatility," Journal of Risk, 5, Winter.

[14] Gyöngy, I. (1986), "Mimicking the One-Dimensional Marginal Distributions of Processes Having an Ito Differential," Probability Theory and Related Fields, 71, 501-516.

[15] Hirsch, F. and G. Lacombe (1999), Elements of Functional Analysis, Graduate Texts in Mathematics, Springer Verlag, Berlin.

[16] Kou, S. G. (2002), "A Jump Diffusion Model for Option Pricing," Management Science, 48, 1088-1101. 
[17] Kou, S.G. and H. Wang (2003), "First Passage Times of a Jump Diffusion Process," Advances in Applied Probability, 35, 504-531.

[18] Madan, D.B., P. Carr, E. Chang (1998), "The Variance Gamma Process and Option Pricing," European Finance Review, 2, 79-105.

[19] Madan, D.B. and M. Yor (2002), "Making Markov Martingales Meet Marginals," Bernoulli, 8, 509-536.

[20] Meyer, P. (1976), "Un Cours sur les Intégrales stochastiques," Séminaire de Probabilités X, Lecture Notes in Mathematics, 511, Springer-Verlag, Berlin.

[21] Sato, K. (1999), Lévy processes and infinitely divisible distributions, Cambridge University Press, Cambridge.

[22] Yor, M. (1978), "Rappels et Préliminaires Généraux," in: Temps Locaux, Société Mathématique de France, Astérisque, 52-53, 17-22. 